\title{
MATHEMATICAL DESCRIPTION OF AN ASYNCHRONOUS MOTOR WITH THE INDIRECT CONTROL OF THE OUTPUT MECHANICAL VARIABLES
}

\author{
A.V. Glazachev ${ }^{1,}$,, Yu. N. Dementyev ${ }^{1}$, K.N. Negodin ${ }^{1}$, A.D. Umursakova ${ }^{2}$ \\ ${ }^{1}$ National Research Tomsk Polytechnic University, 634050 Tomsk, Russia \\ ${ }^{2}$ Innovative University of Eurasia, Engineering Academy, Pavlodar, Kazakhstan
}

\begin{abstract}
The article gives the mathematical description of an asynchronous motor with the indirect control of the output mechanical variables of an asynchronous motor in the electric drive. To determine the electromagnetic torque and angular velocity of the asynchronous motor in the electric drive the mathematical description is used in which the values are determined by the readings of the motor and easily measured values by means of known in practice devices. The proposed in the article the mathematical description for the indirect measuring the electromagnetic torque and angular velocity of the asynchronous motor in the electric drive does not contain the integral components that introduce the great error into the value of the controlled electromagnetic torque and angular velocity.
\end{abstract}

\section{Introduction}

Nowadays, in all branches of industry the leading position takes a modern asynchronous electric drive of general purpose industrial mechanisms.

In conveyor drives, carriers, feeders, lift mechanisms when transporting liquid for water supply and sewage system of settlements and industrial enterprises [1, 2], the transportation of oil and oil products from the fields to the processing plants [3], etc. The implementation of high-speed control of the electromagnetic torque of the actuating motor and the continuous control of its speed is required.

In the majority of cases the most common speed measurement devices are impulse and tachometer sensors [4-6], cradle and transmission dynamometers, torsion devices, converting apparatus for measuring the torque $[4,7,8]$. These devices can be implemented on the basis of the additional, built-in into the electric motor or mechanically attached to it micromachines of direct or alternating current, as well as other special devices.

These sensors allow to control the mechanical variables of the electric drive with the necessary accuracy, but they have a complicated construction and require careful installation of the sensors for coupling with the rotating parts of the drive, which leads to a low reliability operation. It should be noted that the use of rotor speed sensors provides a high quality and relatively simple control of the asynchronous motor. However, the presence of these sensors significantly worsens the performance of the electric drive, and their use may not be possible under the terms of the functioning of the drive.

In this regard, the industrial enterprises widely demand the devices and methods that allow to carry out the continuous control of the electromagnetic torque and angular velocity of the general-purpose industrial mechanisms in the technological process, the monitoring of the workload of actuating motors, to maintain the motor speed within specified limits or the process variable in accordance with the technical and technological requirements, when the necessary information is calculated by indirect methods.

\section{Setting of the task and the objective}

And unlike the existing ones [9-16] the proposed in the article the mathematical description to reduce the error in determining the variable of the electromagnetic torque and angular velocity does not contain the integral components

\footnotetext{
${ }^{\mathrm{a}}$ Corresponding author: alexg@tpu.ru
}

This is an Open Access article distributed under the terms of the Creative Commons Attribution License 4.0, which permits unrestricted use, distribution, and reproduction in any medium, provided the original work is properly cited. 


\section{Mathematical description of the asynchronous motor}

To determine the electromagnetic torque and the angular velocity of the asynchronous motor the mathematical description is used [14], wherein their values are determined according to the readings of the motor and easily measurable values using the known in practice devices.

$$
\left\{\begin{array}{l}
M(t)=\sqrt{3} p_{n}\left(i_{a}(t) \int\left[u_{b}(t)-R_{s} i_{b}(t)\right] d t-i_{b}(t) \int\left[u_{a}(t)-R_{s} i_{a}(t)\right] d t\right) \\
\omega(t)=\omega_{\mathrm{m}}(t)\left[1+\Delta \omega_{\mathrm{int}}(t)+\Delta \omega_{\mathrm{dif}}(t)\right]
\end{array}\right.
$$

where $\omega_{\mathrm{m}}(t)=\frac{\sqrt{3}\left[u_{a}(t)-\left(R_{s}+R_{r \alpha}^{\prime}\right) i_{a}(t)\right]}{\int\left(R_{s}\left[i_{a}(t)+2 i_{b}(t)\right]-\left[u_{a}(t)+2 u_{b}(t)\right]\right) d t-L_{\beta}\left[i_{a}(t)+i_{b}(t)\right]}-$ measured the instantaneous value of the angular velocity; $\Delta \omega_{\mathrm{int}}(t)=\frac{\int\left[u_{a}(t)-R_{s} i_{a}(t)\right] d t}{T_{r}^{\prime}\left[u_{a}(t)-\left(R_{s}+R_{r \alpha}^{\prime}\right) i_{a}(t)\right]}-$ the dynamic integral component of the relative value of the angular velocity; $\Delta \omega_{\mathrm{dif}}(t)=\frac{L_{\beta}\left(d i_{a}(t) / d t\right)}{u_{a}(t)-\left(R_{S}+R_{r \alpha}^{\prime}\right) i_{a}(t)}$-the dynamic differential component of the relative value of the angular velocity; $R_{r \alpha}^{\prime}=\alpha R_{r}^{\prime}$ - the active reduced resistance of the rotor winding, taking into account $\alpha$ coefficient; $R_{r}^{\prime}$ - the active reduced resistance of the rotor; $\alpha=L_{S} / L_{r}^{\prime}$ - the coefficient equal to the ratio of the total inductance of the stator winding $L_{S}$ to the reduced total inductance of the rotor winding $L_{r}^{\prime} ; L_{\beta}=\beta L_{\mu}-L_{S}$ - the inductance with account of $\beta$ coefficient; $L_{\mu}$-the mutual inductance of the stator and rotor windings; $\beta=L_{\mu} / L_{r}^{\prime}$-the coefficient equal to the ratio of the mutual inductance $L_{\mu}$ to the reduced total inductance of the rotor winding $L_{r}^{\prime} ; T_{r}^{\prime}=L_{r}^{\prime} / R_{r}^{\prime}$ - the rotor time constant.

\section{Research methods}

The presence of the integral components available in the mathematical description of the asynchronous motor with the indirect control of the system of equations (1), leads to the errors, when determining the values of the currents and voltages, and can lead to the accumulation of significant errors in the values of the controlled electromagnetic torque and angular velocity of the asynchronous motor. Therefore, in the calculation of the integral components of the equation system (1) we use the known mathematical expressions for the analytic signals

$$
u_{a}(t)=\sum_{k=0}^{\infty} A_{k} \sin \left(k \omega t+\varphi_{k}\right)+j \sum_{k=0}^{\infty} A_{k} \cos \left(k \omega t+\varphi_{k}\right)
$$

Then,

$$
\begin{gathered}
\int_{0}^{1 / f}\left[u_{a}(t)-z i_{a}(t)\right] d t=\int_{0}^{1 / f} \tilde{U}_{a}(t) d t= \\
=\int_{0}^{1 / f}\left\{\sum_{k=0}^{\infty} A_{k}\left(\sin k \omega^{*} t+\varphi_{k}^{*}+j \cos k \omega^{* *} t+j \varphi_{k}^{* *}\right)\right\} d t- \\
=\int_{0}^{1 / f}\left\{\sum_{k=0}^{\infty} B_{k} \sin k \omega^{* *} t+\varphi_{k}^{* *}+j \cos k \omega^{* *} t+j \varphi_{k}^{* *}\right\} d t .1
\end{gathered}
$$

Next, using Euler's formula we can pass from the trigonometric functions to exponential ones [17-20].

In general view, the integrand has the following form. 
Then in general terms, we get that the integrand has the form

$$
\tilde{U}_{a}(t)=\sum_{k=-\infty}^{+\infty} C_{k} e^{j \frac{\pi k t}{l}}, 1
$$

where the coefficient $C_{k}$ can be defined as follows

$$
C_{k}=\frac{1}{2 l} \int_{-l}^{l} u(t) e^{-j \frac{\pi k}{l}} d t
$$

i.e. the harmonic of $e^{j k t}$ form gives the wave number spectrum with the amplitude

$$
C_{k}=\frac{1}{2 l} \int_{-l}^{l} u(t) e^{-j \frac{\pi k}{l}} d t
$$

Then we can use Fourier transform, which for the signal

$$
\tilde{U}_{a}(t)=u(t) e^{j k t n},
$$

where $k=-\frac{\pi}{l}$

is of the form of

$$
\tilde{U}(j \omega)=u \int_{0}^{\infty} e^{-(a+j \omega) t} d t=\frac{u}{a+j \omega}=u(\omega) e^{j \varphi(\omega)}
$$

Since the function is defined only for $t \succ 0$, it is also possible to use sine and cosine of Fourier transform.Fourier integral approach is usually written in the form of

$$
u(t) \approx \int_{0}^{N}(a(\omega) \cos \omega t+b(\omega) \sin \omega t) d t=\sum_{k=0}^{\infty}\left(C_{k} e^{-k t \omega}+C_{-k} e^{k t \omega}\right)=\sum_{k=0}^{\infty}-C_{k}^{*} \sin \omega t
$$

Thus, finally the integral component transfers into its orthogonal pair, representing the sum of the series. The calculations of $C_{k}$ coefficients are made according to the formulas presented in $[17,19]$.

$$
C_{k}=\frac{1}{2 l} \int_{-l}^{l} u(t) e^{-j \frac{\pi k t}{l}} d t
$$

Using Parseval's formula and the inversion we get $f(t)=e^{-j \frac{\pi k t}{l}}$, denoting $-j \frac{\pi k t}{l}=a$, we get

$$
\begin{gathered}
f(t)=e^{-a t}, \\
u(t)=\frac{1}{a+j \omega}=u(\omega) e^{-j \varphi(t)},
\end{gathered}
$$




$$
\begin{gathered}
g(t)=1, x=x^{0}, v=1, \\
\int_{+0}^{l} u(t) e^{-a t} d t=\frac{2^{0} \Gamma\left(0+\frac{1}{2}\right)}{\sqrt{\pi}\left(a^{2}+1\right)^{0+\frac{1}{2}}}=\frac{\Gamma\left(\frac{1}{2}\right)}{\sqrt{\pi}\left(a^{2}+1\right)^{\frac{1}{2}}}, \\
C_{k}=\frac{1}{2 l} \frac{\Gamma\left(\frac{1}{2}\right) l}{\sqrt{\pi}\left(\pi^{2} j^{2} k^{2}+l^{2}\right)}=\frac{\Gamma\left(\frac{1}{2}\right)}{2 \sqrt{\pi}\left(\pi^{2} j^{2} k^{2}+l^{2}\right)}, \\
k=-\frac{\pi}{l} ; l=1 ; k=-\pi .
\end{gathered}
$$

From the above it follows

$$
\begin{aligned}
& M(t)=\sqrt{3} p_{n}\left[i_{a}(t) \int\left[u_{b}(t)-R_{s} i_{b}(t)\right] d t-i_{b}(t) \int\left[u_{a}(t)-R_{s} i_{a}(t)\right] d t\right]= \\
& \sqrt{3} p_{n}\left\{i_{a}(t)\left\{\frac{\Gamma\left(\frac{1}{2}\right)}{\sqrt{\pi}\left(\pi^{2} j^{2} \pi^{2}+1^{2}\right)^{\frac{1}{2}}}-R_{S} \frac{\Gamma\left(\frac{1}{2}\right)}{\sqrt{\pi}\left(\pi^{2} j^{2} \pi^{2}+1^{2}\right)}\right\}-\right. \\
& \left.-i_{b}(t)\left\{\frac{\Gamma\left(\frac{1}{2}\right)}{\sqrt{\pi} \sqrt{1-\frac{\pi^{4}}{4}}}-R_{S} \frac{\Gamma\left(\frac{1}{2}\right)}{\sqrt{\pi} \sqrt{1-\frac{\pi^{4}}{4}}}\right\}\right)= \\
& =\sqrt{3} p_{n}\left(i_{a}(t)\left\{\frac{\sqrt{\pi}}{\sqrt{\pi} \sqrt{1-\pi^{4}}}-R_{S} \frac{\sqrt{\pi}}{\sqrt{\pi} \sqrt{1-\pi^{4}}}\right\}-i_{b}(t)\left\{\frac{\sqrt{\pi}}{\sqrt{\pi} \sqrt{1-\frac{\pi^{4}}{4}}}-R_{S} \frac{\sqrt{\pi}}{\sqrt{\pi} \sqrt{1-\frac{\pi^{4}}{4}}}\right\}\right)= \\
& =\sqrt{3} p_{n}\left(i_{a}(t)\left(\frac{1}{\sqrt{1-\pi^{4}}}-R_{S} \frac{1}{\sqrt{1-\pi^{4}}}\right)-2 i_{b}(t)\left(\frac{1}{\sqrt{4-\pi^{4}}}-R_{S} \frac{1}{\sqrt{4-\pi^{4}}}\right)\right)= \\
& =\sqrt{3} p_{n}\left[\left(1-R_{S}\right) e^{j \frac{\pi}{2}}\left\{2 i_{b}(t) \frac{1}{\sqrt{\pi^{4}-4}}\right\}-i_{a}(t) \frac{1}{\sqrt{\pi^{4}-1}}\right]
\end{aligned}
$$

For $\omega_{m}(t)$

$$
\int\left(R_{s}\left[i_{a}(t)+2 i_{b}(t)\right]-\left[u_{a}(t)+2 u_{b}(t)\right]\right) d t=
$$




$$
\begin{aligned}
& =\left(R_{S}-1\right)\left\{\frac{1}{\sqrt{1-\pi^{4}}}-\frac{2}{\sqrt{4-\pi^{4}}}\right\}= \\
& =\left(R_{S}-1\right) e^{j \frac{\pi}{2}}\left\{\frac{2}{\sqrt{\pi^{4}-1}}-\frac{1}{\sqrt{\pi^{4}-4}}\right\}= \\
& \omega_{\mathrm{m}}(t)=\frac{\sqrt{3}\left[u_{a}(t)-\left(R_{s}+R_{r \alpha}^{\prime}\right) i_{a}(t)\right]}{\left(R_{S}-1\right) e^{j \frac{\pi}{2}}\left\{\frac{2}{\sqrt{\pi^{4}-1}}-\frac{1}{\sqrt{\pi^{4}-4}}\right\}-L_{\beta}\left[i_{a}(t)+i_{b}(t)\right]} .
\end{aligned}
$$

For $\Delta \omega_{\text {int }}(t)$

$$
\begin{aligned}
& \int\left[u_{a}(t)-R_{S} i_{a}(t)\right] d t=\frac{1}{\sqrt{1-\pi^{4}}}-\frac{2 R_{S}}{\sqrt{4-\pi^{4}}}=e^{j \frac{\pi}{2}}\left\{\frac{2 R_{S}}{\sqrt{\pi^{4}-1}}-\frac{1}{\sqrt{\pi^{4}-4}}\right\} \\
& \omega_{\text {int }}(t)=\frac{e^{j \frac{\pi}{2}}\left\{\frac{2 R_{S}}{\sqrt{\pi^{4}-1}}-\frac{1}{\sqrt{\pi^{4}-4}}\right\}}{T_{r}^{\prime}\left[u_{a}(t)-\left(R_{S}+R_{r \alpha}^{\prime}\right) i_{a}(t)\right]} .
\end{aligned}
$$

\section{Results}

Thus, in its final form with account of the above-mentioned the mathematical description of the asynchronous motor with the indirect control of the electromagnetic torque and angular velocity can be written as follows

$$
\left\{\begin{array}{l}
M(t)=\sqrt{3} p_{n}\left[\left(1-R_{s}\right) e^{j \frac{\pi}{2}}\left\{2 i_{b}(t) \frac{1}{\sqrt{\pi^{4}-4}}-i_{a}(t) \frac{1}{\sqrt{\pi^{4}-4}}\right\}\right], \\
\omega(t)=\omega_{\mathrm{m}}(t)\left[1+\Delta \omega_{\mathrm{int}}(t)+\Delta \omega_{\mathrm{dif}}(t)\right]
\end{array}\right.
$$

where

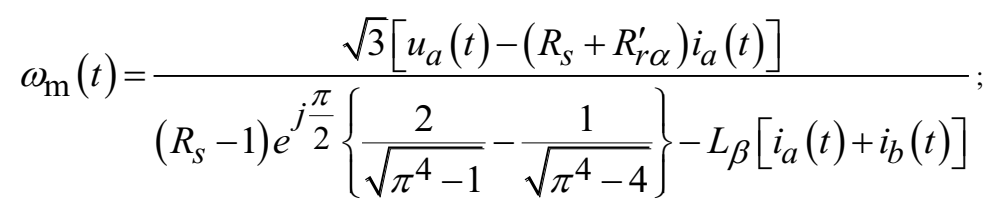

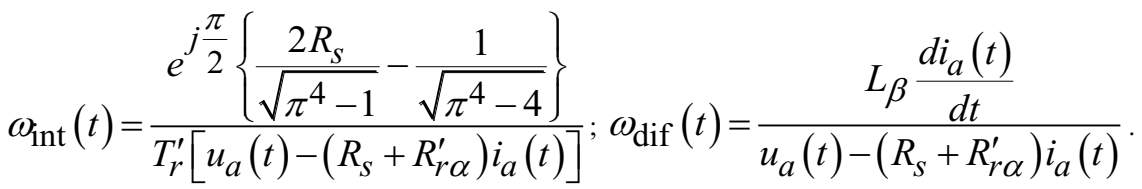

\section{Conclusion}

As a result of the foregoing, the following conclusions can be made: 


\section{EPJ Web of Conferences}

1. It is determined that the proposed analytical dependencies of the mathematical description of the asynchronous motor (AM) with the indirect control of the output mechanical variables allow to calculate the values and continuous monitoring of the electromagnetic torque and angular velocity in the electric drive.

2. To improve the quality of control of the output mechanical variables it is proposed to eliminate the integral component by the transition to an orthogonal pair using Fourier, Euler and Parseval transformation in the mathematical description of the asynchronous motor with the indirect control of the electromagnetic torque and angular velocity of the asynchronous motor.

The research has been performed as a part of the government assignment "Nauka”, project №3852.

\section{References}

1. N.N. Abramov, Water supply (Stroyizdat, 1974)

2. B.S. Leznov. Energy conservation and a variable drive in pump units (Energoatomizdat, 2006).

3. V. B. Galeev. Main oil pipelines (Nedra, 1988)

4. M.B. Leitman. Automatic measuring the output parameters of electric motors (Energoatomizdat, 1983)

5. L.B. Massandilov. Control of rotation speed of asynchronous motors (Energia, 1978)

6. A.Ya. Tun. The systems of speed control of an electric drive (1984)

7. S.D. Levintov. Non-contact magnetoelastic torque sensors (Energoatomizdat, 1984)

8. Yu. I. Rybalchenko. Magnetoelastic torque sensors (Machinostroyenie, 1981)

9. Yu.N. Dementyev, A.D. Umurzakova, V.Yu. Melnikov. Patent for invention, Russia № 2525604, Journal, (23), (2014)

10. Yu.N. Dementyev, A.D. Umurzakova, Useful model patent, Russia, № 131874, Journal, (24), (2013)

11. Yu.N. Dementyev, A.D. Umurzakova, Useful model patent, Russia, № 133314, Journal, (28), (2013)

12. Yu.N. Dementye, Dynamics of systems, mechanisms and machines, (1), 393 (2014)

13. Yu.N. Dementyev, Scientific problems of transportation in Siberia and Far East, (1), 270 (2013)

14. Yu.N. Dementyev, Bulletin of Irkutsk State Technical University, (7), 127 (2013)

15. Yu.N. Dementyev, Fundamental studies, (12), 931 (2014)

16. Yu.N. Dementyev, Omsk scientific bulletin, (3), 200 (2013)

17. V.A. Ditkin, Integral transformations and operational calculus (Nauka, 1974).

18. V.M. Verzhbitski, Numerical methods: Mathematical methods and ordinary differential equations (Oniks, 2005)

19. M.S. Krass, Fundamentals of Mathematics and its applications (Delo, 2003)

20. A.V. Panteleev, Management theory in examples and problems (Vysshaya Shkola, 2003) 